J. Membrane Biol. 32, $231-254$ (1977)

(C) by Springer-Verlag New York Inc. 1977

\title{
Kinetics of Permeability Changes Induced by Electric Impulses in Chromaffin Granules
}

\author{
P. Lindner, E. Neumann *, and K. Rosenheck \\ Department of Membrane Research, The Weizmann Institute of Science, \\ Rehovot, Israel and Max-Planck-Institut für Biochemie, D-8033 Martinsried b. München, \\ Germany
}

Received 10 June 1976

Summary. Electric field pulses, ranging in intensity from 20 to $50 \mathrm{kV} / \mathrm{cm}$ and in duration from 10 to $40 \mu \mathrm{sec}$, caused a transient increase in the membrane permeability of chromaffin granules from the bovine adrenal medulla, that led to partial release of granule soluble constituents. This transient permeability change was long-lived, as compared to the pulse duration, and the main part of material efflux occurred after the termination of the pulse. During the latter phase the temporarily increased permeability decayed to its original value, in the absence of the electric field. This indicated that the structural perturbation induced in the membrane was transient and apparently reversible. The release event was characterized by a field-dependent permeability coefficient ranging from $2 \times 10^{-4} \mathrm{~cm} / \mathrm{sec}$ at $30 \mathrm{kV} / \mathrm{cm}$ to $3 \times 10^{-3} \mathrm{~cm} / \mathrm{sec}$ at $50 \mathrm{kV} / \mathrm{cm}$. The resealing process of the membrane could be described by two relaxation times, both of which decreased with increasing field strength. $\tau_{1}$ varied from about $3.0 \mathrm{msec}$ at $30 \mathrm{kV} / \mathrm{cm}$ to less than $2.0 \mathrm{msec}$ at $50 \mathrm{kV} / \mathrm{cm}$, while $\tau_{2}$ varied from about 100 to about $40 \mathrm{msec}$ in the same interval of field strength. The distribution in the degree of filling of granules that had been partially depleted by an electric field pulse indicated that the population could be considered homogeneous with respect to release.

Chromaffin cells in the adrenal medulla synthesize and store the catecholamine hormones, epinephrine and norepinephrine in subcellular organelles called chromaffin granules. The catecholamines (CA) are secreted in response to stimulation of the cell membrane by acetylcholine (Douglas, 1965). The three main constituents of chromaffin granules are CA, ATP and soluble proteins. The internal concentrations of these materials in the intact granule are $0.55 \mathrm{M}, 0.125 \mathrm{M}$ and $0.133 \mathrm{~g} / \mathrm{ml}$, respectively (Hillarp, 1959). If an electric field, applied to a suspension of the granules in isotonic sucrose solution, exceeds a threshold value, catecholamines and ATP are released (Neumann \& Rosenheck, 1972).

* Present Address: Max-Planck-Institut für Biochemie, D-8033 Martinsried b. München, Germany. 
The study of field-induced changes in the intensity of light scattered by the granules, as well as in the fluorescence of a probe incorporated into the granule membrane (Rosenheck, Lindner \& Pecht, 1975) indicated that it is the membrane that controls the optical effects and catecholamine release.

Since release of content of the chromaffin granules must be caused by changes in the membrane structure, the time course of release will reflect the kinetics of structural changes within the membrane phase. A prerequisite towards the understanding of the release mechanism is the knowledge of the kinetics associated with field-induced structural changes. Therefore, suspension of chromaffin granules were subjected to rectangular electric field pulses and the kinetics of the changes occurring in the light transmitted by these suspensions, was analyzed. Apparent permeability coefficients and permeability decay time constants, and their dependence on the intensity and duration of the applied electric field, as well as on the degree of filling of the granules, were derived. The experimental data were correlated with model calculations on lightscattering properties of particles enclosed by a spherical membrane.

\section{Materials and Methods}

\section{Preparation of Granule Suspension}

Chromaffin granules were prepared from bovine adrenal medulla by the method of isotonic sucrose $(0.27 \mathrm{M})$ density gradient centrifugation (Trifaró \& Dworkind, 1970). In order to remove the Ficoll, the granules were washed three times with isotonic sucrose solution.

Empty granules were prepared by hypotonic lysis of full granules in $0.5 \mathrm{~mm}$ Tris buffer, pH7.4. The lysed granules were centrifuged at $25,000 \times \mathrm{g}$ for $25 \mathrm{~min}$ and resuspended in the buffer. This washing procedure was repeated twice.

For the kinetic analysis full or empty granules were suspended in isotonic sucrose solution $(0.27 \mathrm{M}$ sucrose) containing $0.5 \mathrm{~mm}$ Tris buffer, $\mathrm{pH} 7.4$. In every case the optical density (OD) at $366 \mathrm{~nm}$ of the suspensions was between 0.3 and 0.5 for the full granules and about 0.12 for the empty ones.

Partial depletion of granules was investigated on suspensions of initially full granules in isotonic sucrose solution containing $0.5 \mathrm{~mm}$ Tris buffer, $\mathrm{pH} 7.4$, and $10 \mathrm{~mm} \mathrm{NaCl}$.

The concentration of vesicles in such suspensions may be calculated from turbidity measurements. In this study the turbidity of a suspension is expressed as the OD at $366 \mathrm{~nm}$, for $l=1 \mathrm{~cm}$ light path. Optical densities were measured using a Cary 15 spectrophotometer, thermostated at $5^{\circ} \mathrm{C}$. At the wavelength $366 \mathrm{~nm}$ the granule's main constituents, CA, ATP and proteins, have practically no absorption. Under the condition of zero absorbance, the OD is given by Eqs. (9) and (10) (see Discussion). The volume fraction of the suspended granules is

$$
v=2.3 \cdot \frac{4 r}{3 Q_{s}} \cdot \frac{\mathrm{OD}}{l}
$$


With $Q_{s}=3.2 \times 10^{-2}$ and $r=1.1 \times 10^{-5} \mathrm{~cm}$ (Rosenheck et al., 1975) for full granules (see Discussion below), the volume fraction of the granules in the suspension is given by

$$
v=1.1 \times 10^{-3} \cdot \frac{\mathrm{OD}}{l} \text {. }
$$

The volume fraction of the empty granule preparations was $v=1.6 \times 10^{-2} \cdot \frac{\mathrm{OD}}{l}$.

\section{Impulse Methods}

For the kinetic analysis rectangular electric field pulses were applied to the CG suspensions in an electric field jump apparatus (E-jump). This instrument (Ilgenfritz, 1966; Grünhagen, $1974 a, b$ ) generates rectangular pulses adjustable in voltage $V$ between 5 and $50 \mathrm{kV}$ and in duration between 6 and $150 \mu \mathrm{sec}$; and detects field-induced changes in the optical properties of the sample. The sample is placed between the two flat electrodes of the sample cell, equipped with quartz windows for optical detection. The electrodes have a distance of $d=0.5 \mathrm{~cm}$. The homogeneous electric field across the sample is given by $E=V / d$. A change in intensity of the light transmitted through the sample cell produces a change in the photocurrent of the detection unit. The corresponding voltage changes are displayed on two oscilloscopes with different time base settings, $5 \mu \mathrm{sec} / \mathrm{cm}$ and $2 \mathrm{msec} / \mathrm{cm}$, respectively; both bases are simultaneously triggered when the field is applied to the sample. All optical measurements on this apparatus were carried out using light at $366 \mathrm{~nm}$. The rise-time of the field is $30 \mathrm{nsec}$ and the decay time of the pulse after switching off is $150 \mathrm{nsec}$. The sample cell in the E-jump apparatus has a volume of $0.4 \mathrm{ml}$ and, when filled with the sample, had a resistance $R=18 \mathrm{k} \Omega$. This resistance is high enough to keep the field across the sample at practically constant intensity $(3 \%$ field decay after $40 \mu \mathrm{sec})$.

Partial release of granular content was induced in a conventional T-jump apparatus. In this apparatus exponentially decaying pulses of adjustable initial voltage $V_{o}$ are applied to suspensions placed between the electrodes of a sample cell, $d=0.6 \mathrm{~cm}$, sample volume $0.4 \mathrm{ml}$. Under the present experimental conditions, the time course of the field change is given by

$$
E(t)=\frac{V_{o}}{d} \exp (-t / R C)
$$

where $R$ is the sample cell resistance and $C$ the capacitance of the discharge capacitor. The resistance of the cell filled with the sample is $700 \Omega$. With $C=50 \mathrm{nF}$, the decay time of the field is $35 \mu$ sec.

Since the suspensions contain electrolytes, Joule heating during the electric impulses gives rise to a transient increase in temperature in the thermostated cell. The temperature increase $\Delta T$ after a rectangular impulse of duration $\Delta t$ is given by

$$
\Delta T=\frac{V^{2} \cdot \Delta t}{4.18 \cdot R \cdot v \cdot C_{p} \cdot \rho}
$$

where $V$ is the voltage applied to the cell electrodes, $R=18 \mathrm{k} \Omega$ is the sample cell resistance, $C_{p}=1 \mathrm{cal} / \mathrm{g}$ deg is the specific heat of the suspension, $v=0.4 \mathrm{ml}$ is the cell volume and $\rho=1.05 \mathrm{~g} / \mathrm{cm}^{3}$ is the density of the vesicle suspension. For a characteristic impulse used in this study, $V=20 \mathrm{kV}, \Delta t=40 \mu \mathrm{sec}$, the temperature will increase by $\Delta T$ $=0.5^{\circ} \mathrm{C}$. The temperature increase accompanying an exponentially decaying electric field of initial intensity $V_{o}$ in a T-jump apparatus is

$$
\Delta T=C \cdot V_{o}^{2} /\left(8.36 \cdot C_{p} \cdot \rho \cdot v\right) .
$$


With $C=5 \times 10^{-8} \mathrm{~F}$ and $V_{o}=20 \mathrm{kV}$, the temperature increase $\Delta T=6^{\circ} \mathrm{C}$. All experiments were carried out at $3( \pm 1)^{\circ} \mathrm{C}$; thus, even a temperature increase of $6^{\circ} \mathrm{C}$ will not cause measurable thermal release, since the rate of this process at temperatures below $15^{\circ} \mathrm{C}$ is extremely small (Smith, 1968). It should be noted that in this manner the T-jump apparatus is used by us as an electric pulse generator, the temperature effect being negligible (Neumann \& Rosenheck, 1972; Rosenheck et al., 1975).

\section{Discontinuous Density Gradient Centrifugation}

To decide whether the homogeneous electric field induces partial release from all the granules or whether a fraction releases completely and the rest remains unaffected, the following experiments were performed. Discontinuous sucrose gradients of four layers $(0.27 \mathrm{M} / 0.8 \mathrm{M} / 1.2 \mathrm{M} / 1.6 \mathrm{M})$ were prepared in $5-\mathrm{ml}$ tubes, the $0.27 \mathrm{M}$ layer containing the granules. Four samples of granules were compared: unpulsed granules, empty granules obtained by hypotonic lysis as described before, granules pulsed at $25 \mathrm{kV} / \mathrm{cm}$ and granules pulsed at $42 \mathrm{kV} / \mathrm{cm}$ in a T-jump apparatus. The tubes with the gradients were centrifuged at $35,000 \mathrm{rpm}$ for $60 \mathrm{~min}$ in an SW 50.1 rotor. The whole procedure was carried out at $4{ }^{\circ} \mathrm{C}$. The material in the bands formed between the various layers was taken out by a syringe and lysed in water. The empty granules were sedimented and washed three times with $5 \%$ trichloroacetic acid, dissolved in $0.1 \mathrm{M} \mathrm{NaOH}$ solution and analyzed for membrane protein, according to the method of Lowry, Rosebrough, Farr and Randall (1951).

\section{Correlation between Turbidity and Granule Content}

The turbidity of a suspension of chromaffin granules changes with a change in the vesicular content, $\mathrm{CA}$, ATP, and proteins. To quantify this relationship, the turbidity of partially depleted granules was measured and compared with the concentration of CA, ATP and protein contained in the vesicles.

After inducing partial release by applying an electric field pulse $(20-50 \mathrm{kV} / \mathrm{cm})$ to aliquots of a granule suspension, the samples were diluted with $0.27 \mathrm{M}$ sucrose solution by a factor of 3 to give a final volume of $2.7 \mathrm{ml}$. After measurement of the turbidity the sample was centrifuged at $25,000 \times \mathrm{g}$ for $15 \mathrm{~min}$. The supernatant was removed and the absorbance measured at $265 \mathrm{~nm}: A_{1}$. The pellet was lysed in $2 \mathrm{ml} 0.001 \mathrm{M}$ Tris buffer, $\mathrm{pH}$ 7.4. This procedure produces empty granules. After sedimentation of the empty granules, the absorbance of the supernatant at $265 \mathrm{~nm}$ was measured: $A_{2}$. According to Lambert-Beer's law, absorbance $(265 \mathrm{~nm})$ is proportional to the concentration of both low molecular weight components $(\mathrm{lm}), \mathrm{ATP}$ and $\mathrm{CA}$, having their absorbance maxima at 258 and $278 \mathrm{~nm}$, respectively. The contribution of released soluble protein to the absorption at $265 \mathrm{~nm}$ is negligible compared to that of the catecholamines and ATP. These techniques permit calculating the degree of filling in terms of low molecular weight component, $f_{l m}^{\prime}$ of the granules pulsed at a field intensity $E$ by the relationship

$$
f_{i m}^{\prime}=\frac{A_{2}(E) \cdot 0.74}{A_{1}(E)+A_{2}(E) \cdot 0.74}
$$

where $A_{1}(E)$ and $A_{2}(E)$ are the absorbances of the first and second supernatants and 0.74 is the volume ratio, i.e. $2.0 / 2.7$. The sedimented empty granules were resuspended in $2.7 \mathrm{ml}$ of $0.27 \mathrm{M}$ sucrose solution and the turbidity of the suspensions was measured.

The protein in the first and second supernatants was precipitated by addition of TCA to a final concentration of $5 \%$. The precipitate was washed twice with a $5 \%$ TCA solution dissolved in $0.1 \mathrm{~N} \mathrm{NaOH}$ and analyzed for protein according to the method of Lowry et al. (1951). From the results of this analysis a degree of filling $f_{p}^{\prime}$ in terms of 
protein content is calculated. $f_{p}^{\prime}$ is defined as the fraction of the total amount of protein retained inside the granule

$$
f_{p}^{\prime}=\frac{m_{p 2}}{m_{p 1}+m_{p 2}}
$$

where $m_{p 1}$ and $m_{p 2}$ are the amounts of protein in the first and second supernatant, respectively.

It is now convenient to define a relative turbidity of the suspension after an impulse $E$ was applied:

$$
F=\frac{\mathrm{OD}(E)-\mathrm{OD}_{e}}{\mathrm{OD}(0)-\mathrm{OD}_{e}},
$$

where $\mathrm{OD}(E)$ is the turbidity of the suspension after a pulse of intensity $E$, and $\mathrm{OD}_{e}$ is the turbidity of the empty granules.

\section{Results}

\section{Density Gradient Centrifugation}

After density gradient centrifugation, chromaffin granules that had not been subjected to electric impulses appeared as a pellet at the bottom of each tube; only a very weak band was seen at the border between the 1.2 and $1.6 \mathrm{~m}$ layers. The empty granule bands appeared at the $0.8 / 1.2 \mathrm{M}$ and the $0.27 / 0.8 \mathrm{~m}$ borders. Granules that were partially depleted by electric impulses redistribute as shown in Table 1.

Table 1. Distribution of membrane protein between the various bands on a discontinuous sucrose density gradient

\begin{tabular}{llllll}
\hline $\begin{array}{l}E \\
(\mathrm{kV} / \mathrm{cm})\end{array}$ & \multicolumn{2}{l}{$\%$} & & $f_{l_{m}}{ }^{\mathrm{a}}$ \\
\cline { 2 - 5 } & Pellet & $1.2 / 1.6 \mathrm{M}$ & $0.8 / 1.2 \mathrm{M}$ & $0.3 / 0.8 \mathrm{M}$ & \\
\hline 0 & 100 & - & - & - & 1 \\
25 & 90 & 10 & - & - & 0.69 \\
47 & $-{ }^{\mathrm{c}}$ & 79 & 21 & - & 0.31 \\
50 & - & 71 & 29 & - & 0.25 \\
\hline Empty $^{\mathrm{b}}$ & & & & & \\
granules & - & - & $+{ }^{\mathrm{d}}$ & + & 0 \\
\hline
\end{tabular}

a Taken from Table 2 for comparison.

b Lysed granules (see Materials and Methods).

c - , no band.

d + , band. 


\section{Turbidity and Material Release}

The experimental data, showing a correlation between a change in the turbidity and material released are shown in Table 2 . It is found that $\sim 35 \%$ of the vesicular content is already released prior to application of electric impulses. This is due to the procedures used for the preparation of the samples and was found to be unavoidable, even under the most gentle handling at low temperatures (ice).

To make the experimental data analytically tractable, we consider the freshly prepared granules (before any electric impulse is applied) as "full" ones and assign to them a degree of filling of unity. This is consistent with the definition of the relative turbidity $F$ [see Eq. (7)], by which the suspension of unpulsed granules is assigned a value of unity. In the fifth and sixth column of Table 2, these operationally defined degrees of filling, $f_{l m}$ and $f_{p}$, are listed as a function of electric field applied. Thus, using

and

$$
f_{l m}=\frac{A_{2}(E)}{A_{2}(0)}
$$

$$
f_{p}=\frac{m_{p 2}(E)}{m_{p 2}(0)}
$$

we may correlate the relative turbidity $F$ with $f_{l m}$ and $f_{p}$, as shown in Fig. 1.

In the case of exponentially decaying electric fields, the threshold intensity for material release was found to be about $20 \mathrm{kV} / \mathrm{cm}$. This value is close to that of $18 \mathrm{kV} / \mathrm{cm}$ found in previous studies under similar experimental conditions (Neumann \& Rosenheck, 1972). The threshold for release of protein is somewhat higher than that for the release of the catecholamine and ATP (see Table 2).

Table 2. Degrees of filling and relative turbidities after impulses at various field intensities $^{\mathrm{a}}$

\begin{tabular}{llllll}
\hline $\begin{array}{l}E \\
(\mathrm{kV} / \mathrm{cm})\end{array}$ & $F$ & $f_{l m}^{\prime}$ & $f_{p}^{\prime}$ & $f_{l m}$ & $f_{p}$ \\
\hline 0 & 1 & 0.64 & 0.63 & 1 & 1 \\
20 & 0.86 & 0.57 & 0.62 & 0.87 & 0.98 \\
25 & 0.705 & 0.45 & 0.51 & 0.68 & 0.80 \\
35 & 0.524 & 0.35 & 0.44 & 0.53 & 0.66 \\
41.5 & 0.403 & 0.205 & 0.24 & 0.31 & 0.38 \\
50 & 0.335 & 0.16 & 0.24 & 0.25 & 0.38 \\
\hline
\end{tabular}

a For definitions see text. 


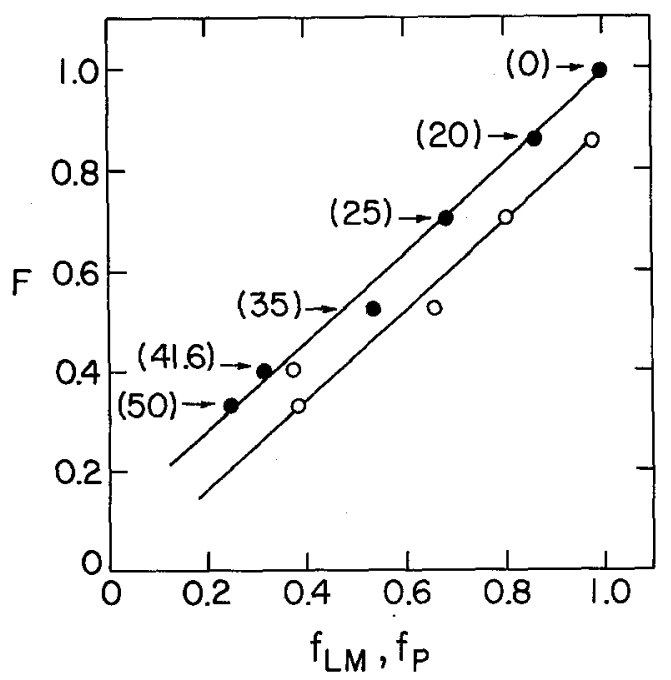

Fig. 1. Relationship between the relative turbidity $F$ and the degrees of filling $f_{p}\left(0^{--}-\right)^{-}$ and $f_{l m}(---)$ of chromaffin granules in $0.27 \mathrm{M}$ sucrose, $0.01 \mathrm{M} \mathrm{NaCl}$ and $1 \mathrm{~mm}$ Tris buffer, $\mathrm{pH} 7.4,5^{\circ} \mathrm{C}$. For definition of $F, f_{p}$ and $f_{I m}$ see text. Numbers in brackets are field intensities in $\mathrm{kV} / \mathrm{cm}$

\section{Kinetics of Changes in Turbidity}

In a series of experiments rectangular electric field pulses were applied to suspensions of chromaffin granules. The electric field always caused an increase in the light transmitted through the suspension.

In Figs. $2 a$ and $3 a$, the changes in the light transmission are shown for suspensions that were subjected to sub- and suprathreshold impulses, respectively. The corresponding transmission changes after the removal of the field are shown in Figs. $2 b$ and $3 b$. The transmission of a sample subjected to an impulse at a subthreshold field intensity $(E<21 \mathrm{kV} / \mathrm{cm})$ increases smoothly and after the external field is removed from the sample, it returns to the original value before the application of the field (see Fig. 2). The changes in the OD corresponding to these changes in transmission are small. An electric impulse of a field intensity $E=$ $16 \mathrm{kV} / \mathrm{cm}$ and a duration of $40 \mu \mathrm{sec}$ causes a transient decrease in the OD of the suspension of about $2 \%$. Previously, a threshold field intensity was correlated with the lower limit for release of material (Neumann \& Rosenheck, 1972). Here, we may redefine the threshold field intensity as the highest value of the field for which the optical changes are still reversible. 


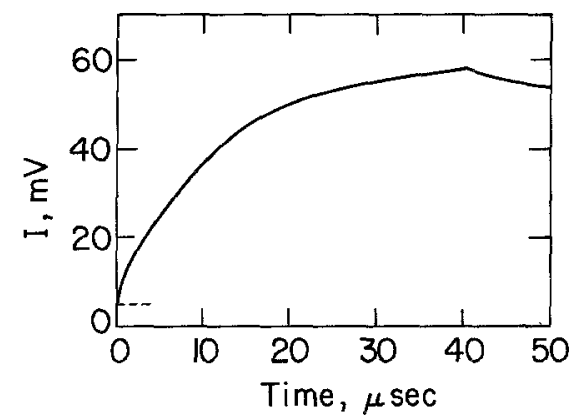

a

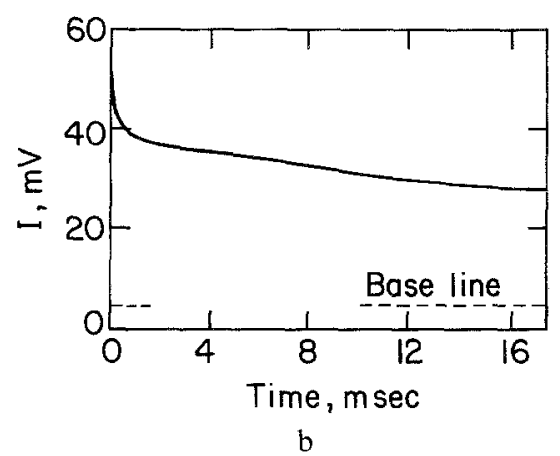

Fig. 2. Light transmittance during and after the application of a subthreshold rectangular pulse ( $E=16 \mathrm{kV} / \mathrm{cm}$, pulse length $\Delta t=40 \mu \mathrm{sec}$ ) to chromaffin granules in $0.27 \mathrm{M}$ sucrose, $0.5 \mathrm{~mm}$ Tris buffer, $\mathrm{pH} 7.4,3.1^{\circ} \mathrm{C}$. $100 \%$ transmittance corresponds to $3.030 \mathrm{~V}:(a)$ During the application of the electric field: before the impulse, the light signal is $I(0)=1.368 \mathrm{~V}$ corresponding to an $\mathrm{OD}$ of 0.346 . Maximum change in $\mathrm{OD}$ induced by the impulse $\triangle O D_{\max }=0.014$. (b) After the impulse: The final value of $\mathrm{OD}$ after the impulse is $\mathrm{OD}(\infty)$ $=0.346$

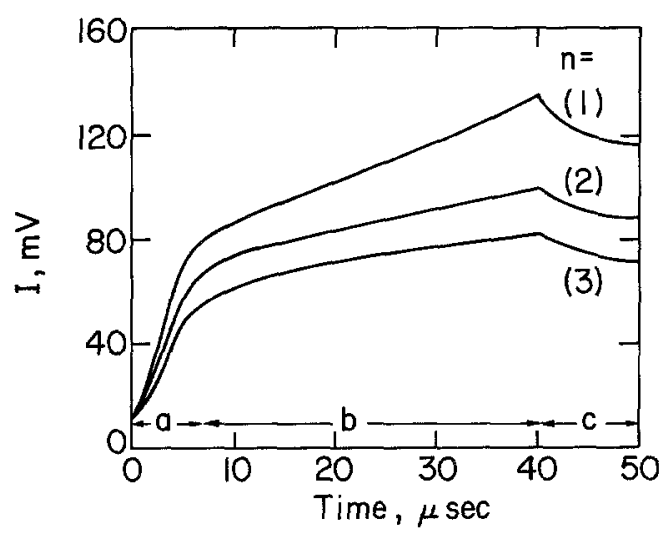

a

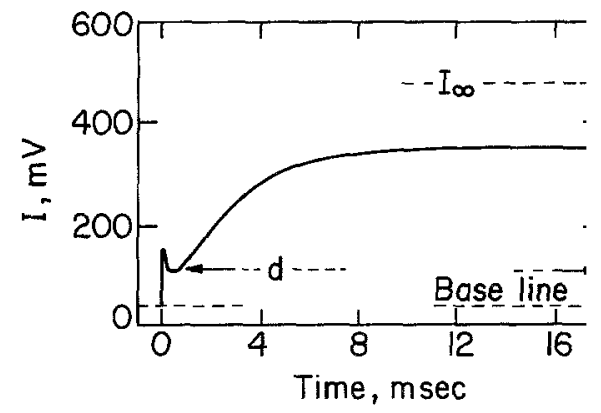

b

Fig. 3. Light transmittance during and after the application of a suprathreshold rectangular pulse $(E=35 \mathrm{kV} / \mathrm{cm}$, pulse length $\Delta t=40 \mu \mathrm{sec})$ to chromaffin granules in $0.27 \mathrm{M}$ sucrose, $0.5 \mathrm{M}$ Tris buffer, $\mathrm{pH} 7.4,3.1^{\circ} \mathrm{C} .100 \%$ transmittance corresponds to $3.030 \mathrm{~V}$; $\lambda=366 \mathrm{~nm}$. (a) During the application of the field, three successive pulses on the same sample

\begin{tabular}{llll}
\hline$n$ & $F(n-1)$ & $\begin{array}{l}I(0) \\
(\mathrm{V})\end{array}$ & $\mathrm{OD}(0)$ \\
\hline 1 & 1 & 1.437 & 0.323 \\
2 & 0.543 & 1.888 & 0.206 \\
3 & 0.426 & 2.026 & 0.175 \\
\hline
\end{tabular}

$F(n-1)$ is the relative turbidity before the $n$th impulse. $I(0)$ and $O D(0)$ are the signal intensity (in V) and OD, respectively, before the impulse at $t=0$. (b) After the first impulse, $n=1$. Final values at $t=\infty: I(\infty)=1.888 \mathrm{~V}, \mathrm{OD}(\infty)=0.206$ 


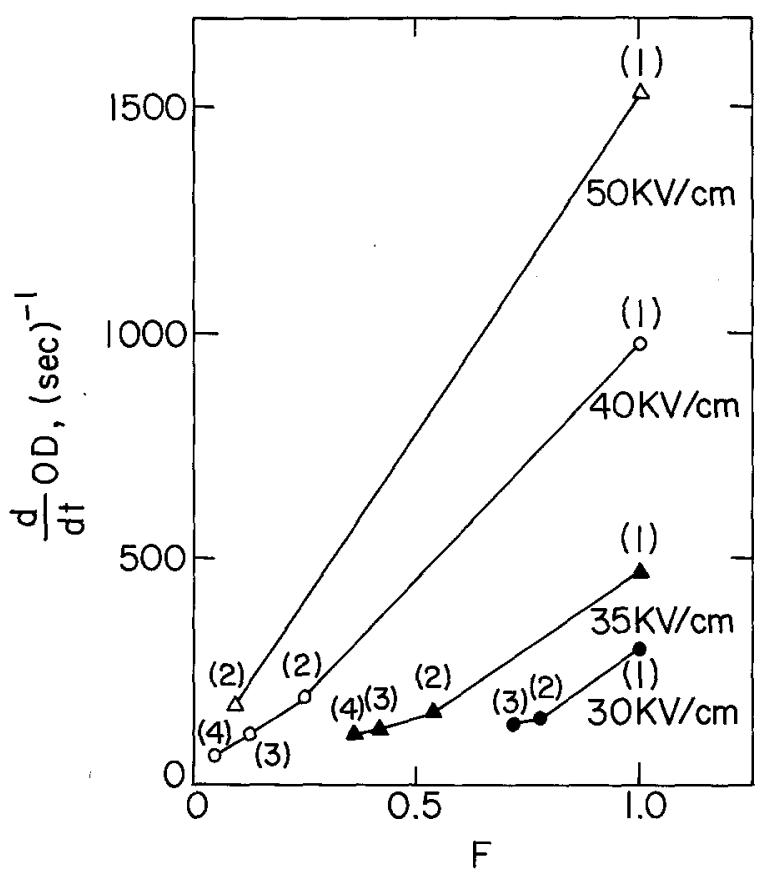

Fig. 4. Rate of change of the OD during phase $b$ (see Fig. $3 a$ ) for a series of successive pulses on the same sample. In brackets: the pulse number. $\Delta t=40 \mu \mathrm{sec}$. Conditions as in Fig. 3

In contrast to subthreshold impulses, the response during a suprathreshold pulse is clearly biphasic (Fig. $3 a$ ). During phase $a$, which is shorter than $10 \mu \mathrm{sec}$, a small but rapid increase in transmittance is observed. This is followed by phase $b$, during which the transmittance increases at an apparently constant rate. This phase remains linear with time, up to measured pulse durations as large as $120 \mu \mathrm{sec}$. After the termination of the impulse, the transmittance first decreases (phase $c$ ) but after about $300 \mu \mathrm{sec}$ it starts to increase again (phase $d$ ) until a new timeindependent level is reached (Fig. $3 b$ ). In Fig. $3 a$ the light transmittance at three successive pulses applied to the same sample is shown as a function of time. Provided that the relative change in transmittance is small, a linear change in transmittance implies also a linear change in OD. Thus,

$$
-\frac{0.434}{I} \cdot \frac{d I}{d t}=\frac{d \mathrm{OD}}{d t} .
$$

In Fig. 4 the rate of change of the OD during phase $b$ is plotted against the relative turbidity at zero time before each pulse, for a series of successive pulses on the same sample. 


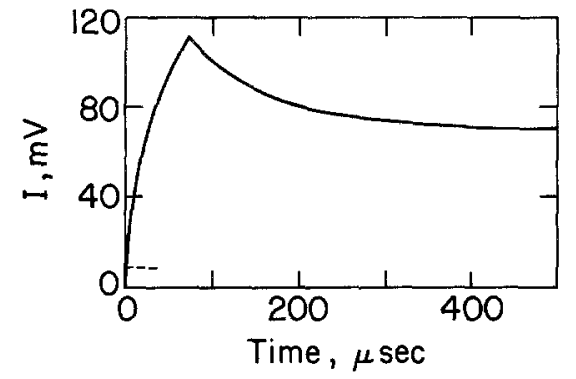

a

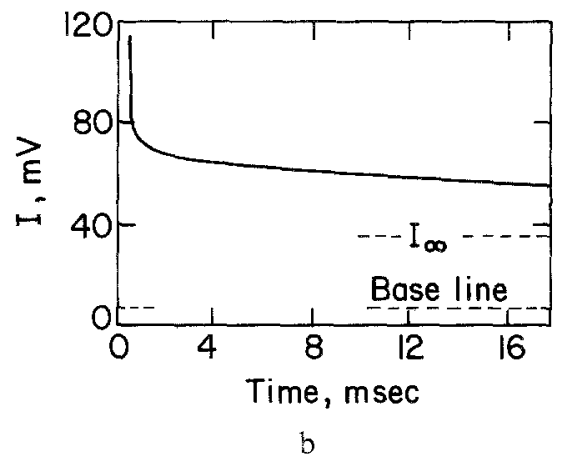

Fig. 5. Light transmittance changes of a suspension of empty chromaffin granules in $0.27 \mathrm{M}$ sucrose, $0.5 \mathrm{M}$ Tris buffer, $\mathrm{pH} 7.4,3.1^{\circ} \mathrm{C}$, during and after the application of a rectangular impulse $(E=48 \mathrm{kV} / \mathrm{cm}) . \Delta t=40 \mu \mathrm{sec}$. $100 \%$ transmittance corresponds to $3.030 \mathrm{~V} ; \lambda=366 \mathrm{~nm}$. (a) During the application of the field $I(0)=2.363 \mathrm{~V} ; \mathrm{OD}(0)=0.105$.

(b) After the impulse. $I(\infty)=2.400 \mathrm{~V} ; \mathrm{OD}(\infty)=0.098$

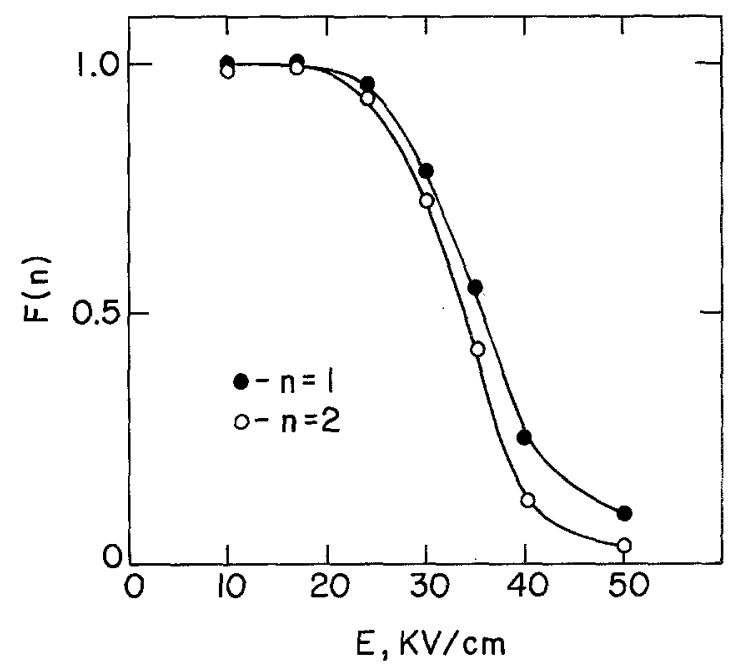

Fig. 6. The relative turbidity of a suspension of full chromaffin granules pulsed at various field intensities, after the first pulse, $n=1(-\bullet)$ and after the second pulse, $n=2$ $(\mathrm{O}-\mathrm{O})$ on the sample. Suspending medium: see legend to Fig. 3

Empty granules behave differently. Subthreshold pulses cause a transient transmittance change that is practically reversible. This behavior is similar to the response to subthreshold pulses of full granules. Suprathreshold pulses applied to empty granules, e.g. $E=40 \mathrm{kV} / \mathrm{cm}$ and pulse length, $\Delta t=40 \mu \mathrm{sec}$, cause transmittance changes that decay to a final value which is about $2 \%$ higher than that before the pulse. This 


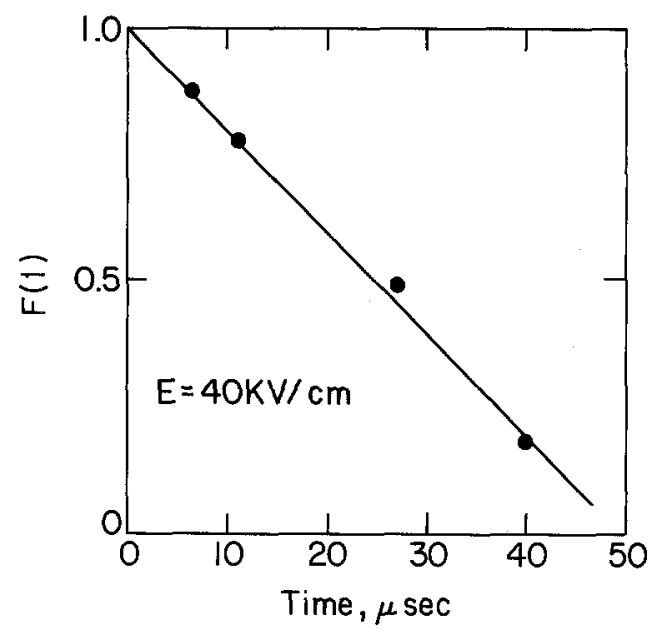

Fig. 7. Dependence of the relative turbidity after the first pulse, $F(1)$, of a suspension of full chromaffin granules on the pulse duration. $E=40 \mathrm{kV} / \mathrm{cm}$. Other conditions as in Fig. 3

irreversible change may be considered small, when compared to the $75 \%$ increase in transmittance caused by the same pulse in suspensions of full granules. The small change of $2 \%$ may reflect release from a small fraction of granules which resisted the lysis procedure, or it may be caused by small very long-lived changes in the membrane structure (see Fig. 5).

In Fig. 6, the relative turbidity $F$, after application of a first and a second pulse, respectively, is plotted as a function of the field intensity $E$, $\Delta t$ being $40 \mu \mathrm{sec}$ in both cases. The threshold intensity found for the appearance of irreversible changes in turbidity is about $21 \mathrm{kV} / \mathrm{cm}$ under the given experimental conditions. An impulse of $16 \mathrm{kV} / \mathrm{cm}$ did not cause irreversible changes in turbidity, even if the pulse duration was $180 \mu \mathrm{sec}$.

In Fig. 7, the relative turbidity, after the first pulse, $F(1)$ is plotted versus the pulse length. We see that $F(1)$ drops linearly with increasing pulse length.

\section{Discussion}

\section{Partial Release}

The data in Table 1 suggest that granules pulsed at suprathreshold intensities have a density intermediate between the densities of full and empty granules. Since the density of the granules is proportional to 
content of catecholamine, ATP and protein, we conclude that partial release of these components was induced by the electric field. The alternative that, depending on a distribution of sensitivities with respect to the electric field, a fraction of the granules is emptied while the others remain full, is not consistent with the experimental data. These results, however, do not imply that the whole granule population releases exactly to the same extent. In fact, a fresh preparation of granules is not homogeneous with respect to density (unpublished results). On a continuous density gradient granules from a fresh preparation distribute between 1.6 and $2.2 \mathrm{M}$ sucrose. The data in Table 1 suggest that the response to the external fields is not uniform. The granule population presumably is heterogeneous both with respect to threshold intensity and extent of release. The coarse stepwise gradient used in this investigation is not suitable for detailed study of the variability in these parameters. However, the variability is small enough to assign, after the pulse, to the granule population representative average degrees of filling, $f_{l m}^{\prime}$ and $f_{p}^{\prime}$, (see Materials and Methods) which depend on the pulse intensity $E$, the pulse number $n$, and the pulse length $\Delta t$.

\section{Processes Leading to Changes in Turbidity}

The turbidity of suspensions at a wavelength $\lambda$ outside of an absorption band depends on the light-scattering properties of the suspended particles. Expressed as OD the turbidity is given by

$$
\mathrm{OD}(\lambda)=\log _{10} \frac{I_{0}(\lambda)}{I(\lambda)}=0.434 \mathrm{Nl} C_{s}(\lambda)
$$

where $I_{0}$ and $I$ are the incident and transmitted light intensities, respectively, $N$ is the number of particles per unit volume, $l$ is the light path length and $C_{s}(\lambda)$ is the scattering cross-section of the particle at wavelength $\lambda$.

$C_{s}(\lambda)$ depends on the individual granule properties and is given by

$$
C_{s}(\lambda)=Q_{s}(\lambda) \cdot S
$$

where $Q_{s}$ is the scattering efficiency and $S$ the projected area, perpendicular to the incident beam (van de Hulst, 1957). The correlations shown in Fig. 1 are the basis for the use of the transmittance at $366 \mathrm{~nm}$ as the quantity by which the time course of the release process is 


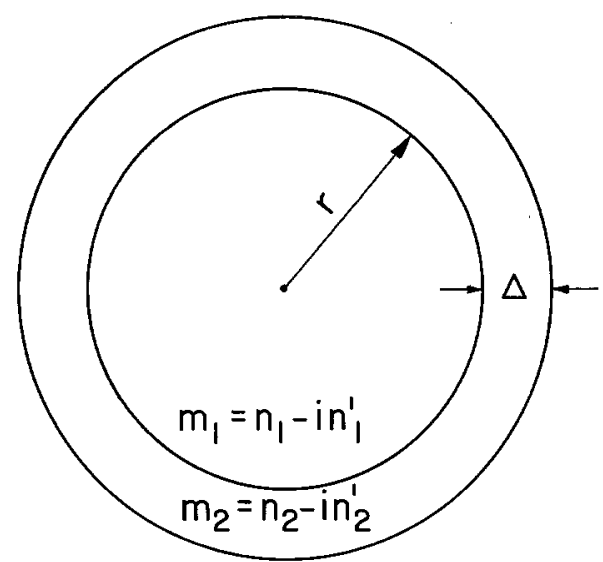

Fig. 8. Parameters of the model for the scattering calculations

followed. As previously noted, release of protein was not always observed concomitantly with release of CA and ATP. Since in the present experiments release of protein essentially parallels the release of the low molecular weight material, we assume that decrease in turbidity, expressed in terms of $F$, corresponds to loss of all three components stored in the granules. (Since the OD and the degrees of filling were measured long after the pulse had decayed, the linear correlation represented in Fig. 1, is strictly valid only for suspensions at equilibrium.)

For the interpretation of the short-term changes in light transmittance during and after the pulse, i.e. before the system re-equilibrates, changes of the scattering properties due to other processes, besides reduction in the degree of filling, must be considered. For the purpose of the light-scattering calculations, chromaffin granules can be approximated by a homogeneous core of radius $r$ and refractive index $m_{1}$ relative to the suspending medium (Fig. 8), bounded by a homogeneous spherical shell of thickness $\Delta$ and of refractive index $m_{2}$, relative to the suspending medium. For this model, Mie type calculations (Fenn \& Oser, 1965) yield exact solutions of the scattering problem in terms of the parameters $m_{1}, m_{2}, \Delta$ and $r$. Since the wavelength of the light used in the measurements is far from the absorption bands of the chromaffin granules, only the real parts of the refractive indices, $n_{1}$ and $n_{2}$, have to be considered (see Fig. 8). The scattering efficiencies $Q_{s}$ computed for parameter ranges relevant to the chromaffin granules are shown in Fig. $9 a-c$.

The data in these Figures can be used to estimate the trends in the changes in turbidity (or light transmittance) due to processes which will affect the membrane or core parameters. 

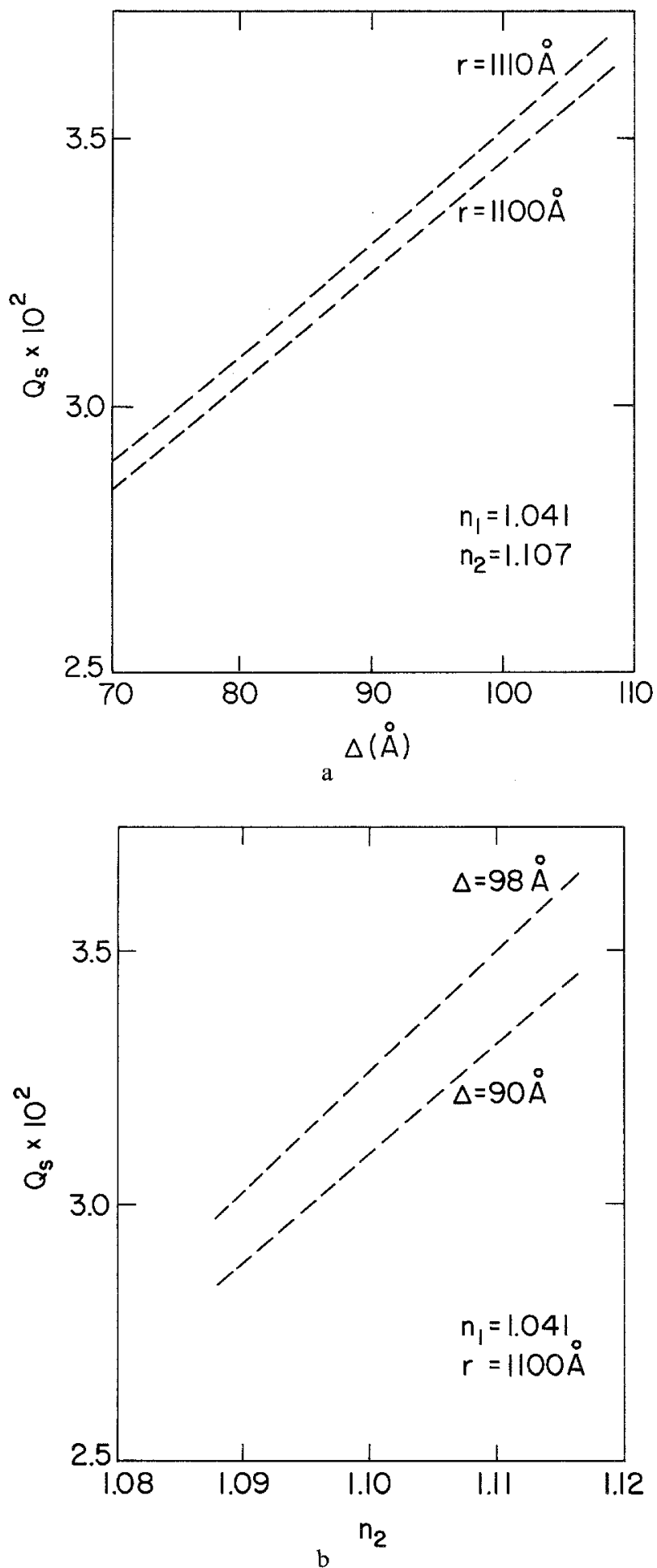

Fig. 9. Dependence of the scattering efficiencies on $(a)$ shell thickness $A,(b)$ membrane refractive index $n_{2},(c)$ core refractive index $n_{1}$ 


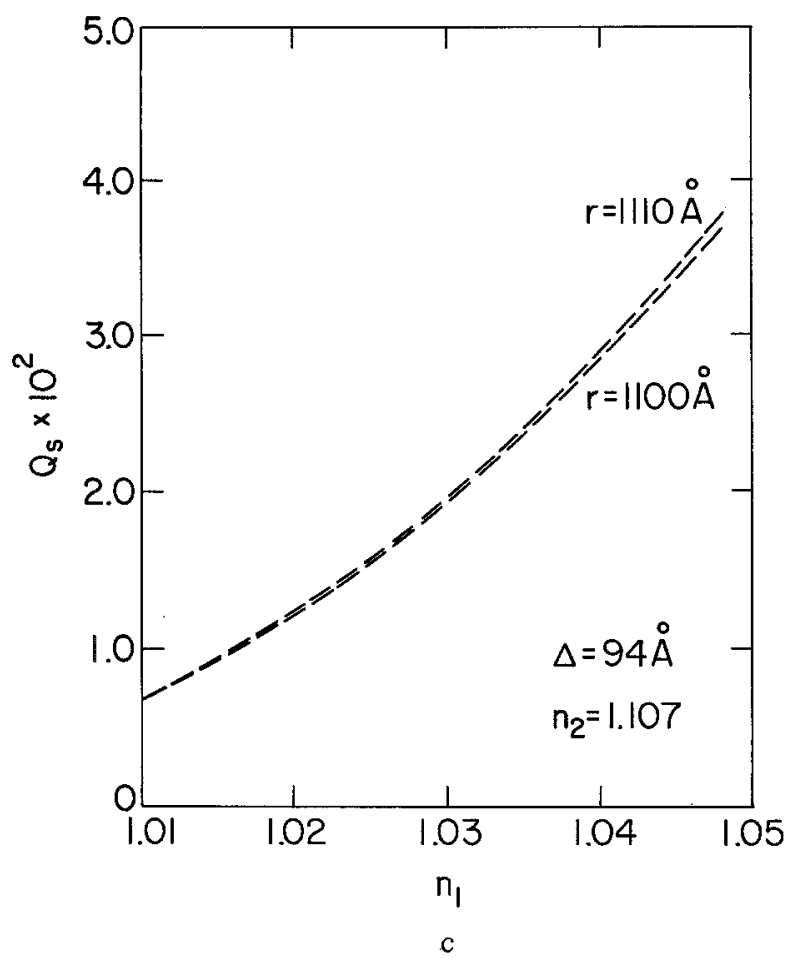

The following processes may be considered which presumably do not cause serious changes in the shape of the granules.

a) Release of soluble material from the granule. Release of core material and solution influx reduces the real refractive index $n_{1}$ of the core. Since, for the parameter range chosen, $\partial Q_{s} / \partial n_{1}>0$ (Fig. 9), the OD of the suspension will decrease provided that other parameters remain unchanged. This is the basis of the correlation shown in Fig. 1. It should be noted that this process cannot be responsible for the transient change in turbidity induced by subthreshold pulses since no material is released under these conditions.

From the data of Smith (1968) we calculate the concentration of soluble protein and low molecular weight components $(\mathrm{CA}+\mathrm{ATP})$ in the core to be 0.133 and $0.173 \mathrm{~g} / \mathrm{cm}^{3}$, respectively. Assuming that the refractive index increments of these materials do not differ appreciably, the contributions of the protein to $n_{1}$ and to the scattering properties are not negligible. This supports the assumption that the permanent changes in OD after the pulse are due to efflux of both protein and low molecular weight material. 
b) Penetration of water from the medium into the membrane. If the membrane structure is disorganized by the action of the external field, influx of external solution will dilute the core. As already mentioned water entrance into the membrane will reduce its refractive index. Since $\partial Q_{s} / \partial n_{2}>0$ (see Fig. 9) we expect a decrease in OD of the suspension. Among the possible mechanisms which may initiate a disorganization of the membrane is counterion polarization (Neumann \& Rosenheck, 1972). For the present experimental conditions of ionic strength, the time constant for counterion polarization is $100-150 \mu \mathrm{sec}$. The light-scattering changes, however, have much smaller time constants (see Figs. $2 a$ and $3 a$ ). Thus it appears improbable that counterion polarization is a main contribution to the optical changes which are assumed to indicate the triggering of the structural changes in the granule membrane. Without doubt there will be interactions of the electric field with surface charges and dipolar groups of the membrane. The normal component of the instantaneous effective field generated on the surface of the membrane on application of the external field tends to displace charges on the membrane, pulling them outwards or inwards, according to their sign. If the value of this component of the effective field exceeds the attractive forces stabilizing the membrane structure, local disorder will be created in the membrane. The random lateral motion of charged species in the membrane may similarly be affected by the tangential component of the effective field. The time scale for small motions of charged species or dipolar groups of components of the membrane surface seems to us to be consistent with a rapid response to external field changes.

c) Thinning of the membrane. Decrease in the thickness of the membrane is expected to precede a possible dielectric breakdown of the membrane arising from electrocompressive forces (Crowley, 1973; Zimmerman, Pilwat \& Riemann, 1974). Although this process does not occur isotropically we may describe it by an average change in the thickness of the membrane. If electrically induced compression is the dominant factor, the OD of the suspension is changed by changes of both the geometrical cross-section $S$ and the scattering efficiency $Q_{s}$. The relative change in the scattering cross-section is derived from Eq. (10):

$$
\frac{d C_{s}}{C_{s}}=\frac{d S}{S}+\frac{d Q_{s}}{Q_{s}}
$$


With Eq. (A.4) (see Appendix) it is seen that

$$
\frac{d C_{s}}{C_{s}}=\left[\frac{2 \Delta}{r+\Delta}+\frac{\Delta}{Q_{s}} \frac{d Q}{d \Delta}\right] \frac{d \Delta}{\Delta} .
$$

Since changing the membrane thickness at constant mass and composition implies also a change in refractive index we have

$$
\frac{d Q_{s}}{d \Delta}=\left[\frac{\partial Q_{s}}{\partial \Delta}\right]_{r, n_{1}, n_{2}}+\left[\frac{\partial Q_{s}}{\partial n_{2}}\right]_{r, n_{1}, \Delta} \cdot \frac{d n_{2}}{d \Delta}
$$

where the partial derivatives in this expression are the slopes of the plots in Fig. 9. $d n_{2} / d \Delta$ can be estimated from the Lorentz-Lorenz equation (see Appendix), considering the membrane on average as a homogeneous phase (see above). For the values $r=1100 \AA, \Delta=90 \AA, n_{1}=1.042$ and $n_{2}$ $=1.107$, the calculations give

$$
\begin{gathered}
Q_{s}=3.2 \times 10^{-2} ; \\
{\left[\frac{\partial Q_{s}}{\partial \Delta}\right]_{r, n_{1}, n_{2}}=2.1 \times 10^{-4} \AA^{-1} ; \quad\left[\frac{\partial Q_{s}}{\partial n_{2}}\right]_{r, n_{1}, \Delta}=2.12 \times 10^{-1} .}
\end{gathered}
$$

Using these values and the value for $d n_{2} / d \Delta$, calculated in the Appendix, we obtain for an average compression $d \Delta / \Delta$ of the membrane, a relative change in $C_{s}$ given by

$$
\frac{d C_{s}}{C_{s}}=-2.1 \frac{d \Delta}{\Delta} .
$$

Eq. (11c) shows that the OD will increase as the membrane becomes thinner. The experimental results, however, demonstrate that electric fields always cause a decrease in OD. Consequently, membrane thinning per se cannot account for the observed optical changes. Therefore, if electro-constrictive contributions were involved in the permeability changes monitored by the present optical measurements, they must occur on a time scale $<1 \mu$ sec. It should be noted that although the Lorentz-Lorenz equation is a crude approximation for as complex a phase as a membrane, we use it only to estimate the trends of the light-scattering properties.

d) Swelling of the granule. When water from the suspending medium penetrates the granule and dilutes the core, the value of $n_{1}$ decreases while the value of $r$ increases. This subject was extensively treated by Latimer, Moore and Bryant (1968). For suspensions of particles of diameter and refractive index of chromaffin granules a decrease in OD is 
expected with swelling caused by water penetration. Thus, swelling would predict correctly the direction of the observed optical changes. Since the granules are suspended in an isotonic medium, there is initially no driving force for water influx into the core of the granule. Usually sucrose does not permeate through the chromaffin granule membrane. A driving force could be provided by at least two mechanisms: (a) Thermal effusion. This mechanism could be operative if the heat exchange between the outside medium and the inside of the granule is slow enough so that during the pulse a temperature gradient is established across the membrane. This mechanism was suggested by Tsong and Kingsley (1975) to explain the turbidity changes of red blood cell suspensions subjected to electric field pulses in a T-jump apparatus. In impulse experiments the temperature increase of the sample lasts for at least a few seconds. If we assume that the rate of heat exchange is slow we would expect that the thermal effusion process and with it the swelling and increase in light transmittance will continue after the termination of the pulse. (b) Influx of sucrose. A field-induced permeability increase in the chromaffin granule membrane will cause influx of sucrose. Furthermore, influx of water will follow the influx of sucrose, to keep the internal osmolarity of the granule constant. When the field is removed, the decay of the acquired permeability is not instantaneous. It is therefore expected that also in this case, swelling and the corresponding increase in transmittance will continue for some time after termination of the pulse, although at a lower rate. From Figs. $2 a$ and $3 a$, it is seen that the increase in transmittance starts to decay immediately when the electric field is removed. Therefore, the observed changes in transmittance during the application of sub- or suprathreshold field intensity (Figs. $2 a$ and $3 a$ ) are not due to swelling, but must be directly related to the presence of the field via the process already mentioned in section b. Influx of sucrose and swelling may contribute during phase $d$. If a swelling process would lead to lysis, the barrier for diffusion of core material would collapse, and a break in the oscillogram representing phase $d$ is expected. Since the transmittance changes "smoothly", the membrane apparently remains intact during the efflux of core material occuring in phase $d$.

These considerations may lead to the following interpretation of the observed turbidity changes. The transmittance changes caused by a subthreshold pulse (Fig. 2a) are ascribed to a small change in the membrane organization which allows penetration of solution from the suspending medium into the membrane. Thus, the accompanying changes in light transmittance are mainly due to changes in $n_{2}$. The 
same processes are suggested for phase $a$ during a suprathreshold pulse (Fig. $3 a$ ). Here, after a few $\mu \mathrm{sec}$ the average distance between membrane components may locally increase to such an extent that the membrane becomes permeable to the material stored in the granule.

During phase $b$ the transmittance increases linearly with time. Since the relative changes in transmittance are small, the decrease in OD is also linear with time [see Eq. (8)]. We may ascribe this reduction in OD mainly to efflux of soluble material from the core, causing reduction in $n_{1}$. At least two permeation processes contribute to the efflux occurring in the presence of the field. One contribution is electrophoretic, enhanced by the reduction in membrane resistance at the end of phase $a$. The charged species stored in the granule $\left(\mathrm{CA}^{+1}, \mathrm{ATP}^{-4}\right.$, and the negatively charged proteins), will move in the electric field established through the granule. The second contribution is diffusive. The structural change in the membrane at the end of phase $a$ allows diffusion of stored material through the membrane, along the concentration gradients.

The constant rate of the efflux during phase $b$ implies that the intragranular concentration does not change appreciably during the time interval of the pulse. Although the efflux rates may be high, the pulse length (at most $40 \mu \mathrm{sec}$ ) is too short for a drastic change in the internal concentration. This conclusion is supported by the linear dependence of the release [plotted as a decrease in $F(1)$ ] as a function of the pulse length (Fig. 7). This relationship is linear even for pulse durations for which the degree of filling of the granules is appreciably reduced. If most of the efflux occurred during the pulse, the outflow rate would decrease before the electric field is removed and phase $b$ would not be linear. Furthermore, the observed constant rate of efflux indicates that the permeability coefficient does not change during phase $b$.

Analysis of the time course of the transmittance changes after the pulse (see below) shows that the "diffusive" permeability coefficient increases with the pulse length. This suggests that the permeation process during the pulse is mainly electrophoretic. The electrical resistance of the membrane is not further reduced once the permeation process starts. When the field is removed from the sample, the electrophoretic efflux stops. The rate of the electrophoretic efflux depends on the concentration of the charged species inside the granule. This qualitatively accounts for the decrease in $d \mathrm{OD} / d t$ during phase $b$ in a series of successive pulses on the same sample (see Figs. $3 a$ and 4).

Phase $c$ (Fig. $3 a$ ) represents the beginning of a slow recovery process during which the membrane returns to its normal structure and the 
"diffusive" permeability decreases. During this phase, the transmittance changes are governed by increase in $n_{2}$. This process is slow, and material continues to permeate by diffusion through the membrane. After about 200 to $300 \mu \mathrm{sec}$ the transmittance changes are again determined by $n_{1}$ (phase $d$, Fig. $3 b$ ). $n_{1}$ decreases as the depletion process goes on. The transmittance increases until a new stable level is reached when the permeability has decayed to virtually zero value and the passive efflux stops.

\section{Time Course of the Release Process}

At present it is not possible to resolve the individual time courses of release of the various components stored in the granule. We therefore express the amount of released material in terms of number of moles $n$ of "units of storage complex" containing CA, ATP and protein in a constant ratio. This is justified in our specific case since changes in $f_{p}$ parallel more or less charges in $f_{l m}$ (see Fig. 1).

The amount of material released at time $t$ from spherical granules of radius $r$ is given by

$$
n(t)=J \cdot A \cdot \Delta t+A \int_{t_{p}}^{t} J(t) d t
$$

where the surface area $A$ is $4 \pi r^{2} ; \Delta t=t_{p}-t_{0}$ is the pulse length and $J=A^{-1} \cdot d n / d t$ is the flux, assumed to be constant during the pulse. Consistent with the experimental data, the first term of the right-hand side of Eq. (13) representing the efflux during the pulse length $\Delta t$ is small compared to the second term. Since the material efflux extends over a time interval very much larger than $\Delta t$, we may approximate the total "diffusive" release by the integral as beginning at $t=0$. After the electric pulse is terminated (i.e. during phases $c$ and $d$ ) the "diffusive" permeability coefficient $P(t)$ is assumed to decay according to

$$
P(t)=P_{0} \sum_{i} a_{i} e^{-t / \tau_{i}} ; \quad \sum_{i} a_{i}=1
$$

where $P_{0}$ is the "diffusive" permeability coefficient at the instant of termination of the pulse. The $\tau_{i}$ 's are the time constants of the various processes involved in the decay of the permeability. Neglecting any coupling with fluxes of components of the suspending medium, the 
permeability equation is written

$$
J=-\frac{v_{g}}{A} \frac{d c(t)}{d t}=\left(P_{0} \sum_{i} a_{i} e^{-t / \tau_{i}}\right) \cdot \Delta c(t)
$$

where $v_{g}$ is the volume of the granule and $\Delta c(t)$ the concentration difference of material between the inside and outside at time $t$. For a low volume fraction of the suspended granules, $\Delta c \cong c$, where $c$ is the concentration inside the granule. This holds even for very low degrees of filling. Integration of Eq. (15) yields the time dependence of the degree of filling $f=c(t) / c(0)$, during phase $d$ (see Fig. $3 b$ ). In accordance with Eqs. $(5 b)$ and $(6 b) f$ is defined here for the "storage unit" mentioned above.

To correlate OD with $f$, it is assumed that the transmittance changes during phase $d$ are predominantly controlled by changes in $n_{1}$. Furthermore, the linear relationship established in Fig. 1, for equilibrated suspensions, is assumed to hold already within a very short time interval, i.e. a few milliseconds, after termination of the pulse. Denoting by OD(0) the optical density at $t=0$ and by $\mathrm{OD}_{e}$, the $\mathrm{OD}$ of the empty granule suspension, the combination with the integrated form of Eq. (15) gives

$$
\ln \frac{\mathrm{OD}(t)-\mathrm{OD}_{e}}{\mathrm{OD}(0)-\mathrm{OD}_{e}}=-\frac{3}{r} P_{0} \sum_{i} a_{i} \tau_{i}\left\{1-e^{-t / \tau_{i}}\right\}
$$

For the equilibrated sample $(t \rightarrow \infty)$ after the $n$th pulse, the change in the relative turbidity is given by

$$
\ln \frac{F(n)}{F(n-1)}=-\frac{3}{r} P_{0} \sum_{i} a_{i} \tau_{i} .
$$

The time constants of the processes involved in the "resealing" of the membrane were evaluated from the analysis of the transmittance data after the pulse. The analysis was performed in terms of two processes. The time constant of the slower process can be reliably estimated. However, its relative contribution, $a_{2} /\left(a_{1}+a_{2}\right)$, is probably overestimated since other processes, slower by orders of magnitude, might contribute to the late part of phase $d$. These slow processes could not be followed, due to the instability of the detection system, which limits the time of measurement to $20 \mathrm{msec}$ after the pulse. The transmittance during the early part of phase $d$ is only approximately controlled by changes in $n_{1}$; 
Table 3. Results of the analysis of the transmittance changes after the pulse

\begin{tabular}{lllllllll}
\hline $\begin{array}{l}E \\
(\mathrm{kV} / \mathrm{cm})\end{array}$ & $\begin{array}{l}\text { Pulse } \\
\text { length } \\
(\mu \mathrm{sec})\end{array}$ & $\begin{array}{l}\text { Pulse } \\
\text { number }\end{array}$ & $a_{1}$ & $\begin{array}{l}\tau_{1} \\
(\mathrm{msec})\end{array}$ & $a_{2}$ & \multicolumn{1}{l}{$\begin{array}{l}\tau_{2} \\
(\mathrm{msec})\end{array}$} & $P_{0}$ & $\frac{F(n)}{F(n-1)}$ \\
\hline 30 & 40 & 1 & 0.987 & 3.0 & 0.013 & 96 & $2.2 \times 10^{-4}$ & 0.78 \\
35 & 40 & 1 & 0.989 & 2.2 & 0.011 & 117 & $6.8 \times 10^{-4}$ & 0.550 \\
40 & 40 & 1 & 0.977 & 1.6 & 0.023 & 49 & $2.1 \times 10^{-3}$ & 0.252 \\
50 & 40 & 1 & 0.975 & 1.8 & 0.025 & 36 & $3.1 \times 10^{-3}$ & 0.1 \\
40 & 27 & 1 & 0.980 & 1.9 & 0.020 & 52 & $9.5 \times 10^{-4}$ & 0.496 \\
40 & 40 & 2 & 0.985 & 1.3 & 0.015 & 48 & $1.3 \times 10^{-3}$ & 0.515 \\
\hline
\end{tabular}

during this time interval $n_{2}$ still changes and the total efflux of core material is as yet small. Therefore, the very first points in phase $d$ cannot be used for the evaluation of the time constant for the fast process.

The results of the kinetic analysis are shown in Table 3 . As the pulse intensity increases, the "diffusive" permeability coefficient $P_{0}$ increases and the time constant $\tau_{2}$ decreases. It is evident that release is enhanced by an increase in $P_{0}$, or by increase in time constant for the resealing of the membrane, or both. $P_{0}$ decreases with decreasing degree of filling $F$ by about $40 \%$ from the first to the second pulse, while the time constants $\tau_{1}$ and $\tau_{2}$ remain practically constant. The dependence of $P_{0}, \tau_{1}$ and $\tau_{2}$ on the pulse length could not be analyzed since for pulses of short duration (i.e. $\Delta t<15 \mu \mathrm{sec}$ ), less than $50 \%$ of the total change in OD had taken place at the end of the time interval during which accurate measurements could be made.

In summary, the experimental evidence for partial release presented above suggests that electric field pulses are able to produce long-lived permeability changes, most probably caused by structural changes in the membrane of the chromaffin granule. These changes anneal very slowly, as compared to the duration of the applied fields, but they are basically reversible. Irreversible processes, such as granule destruction, are certainly not dominant in the electric field-induced permeability changes. The release event can be characterized by a field-induced permeability coefficient $P_{0}$ and by two relaxation times $\tau_{1}$ and $\tau_{2}$ related to the resealing process of the membrane. At the present stage of experimentation it is not yet possible to correlate the basic physico-chemical data of in vitro release with mechanisms for neuro-secretory processes, such as the "stimulated" release of hormones from their storage vesicles at the subcellular level. 
P. Lindner gratefully acknowledges an EMBO Fellowship. The experiments reported in this study were carried out at the Max-Planck-Institut für Biophysikalische Chemie, Göttingen. We thank Prof. Manfred Eigen for the generous support of our work. This research was supported in part by grants from the United States-Israel Binational Science Foundation (BSF), Jerusalem, Israel and the Stiftung Volkswagenwerk.

\section{Appendix}

\section{(A) Estimation of $d n_{2} / d \Delta$}

The Lorentz-Lorenz equation is

$$
\frac{n_{2}^{2} n_{0}^{2}-1}{n_{2}^{2} n_{0}^{2}+2}=\frac{4 \pi}{3} \cdot \frac{\sum \alpha_{i} N_{i}}{v_{m}}
$$

where $n_{0}$ is the real refractive index of the medium (relative to vacuum) and $n_{2}$ the real refractive index of the membrane relative to that of the medium; $\alpha_{i}, N_{i}$ are the polarizability and number of molecules, respectively, of component $i$; and $v_{m}$ is the volume of the membrane. Assuming that the compression occurs at constant composition

$$
\frac{d n_{2}}{d v_{m}}=-\frac{n_{2}^{4} n_{0}^{4}+n_{2}^{2} n_{0}^{2}-2}{6 n_{2} n_{0}^{2}} \cdot \frac{1}{v_{m}}
$$

If $\Delta \ll r$, where $r$ is the inner radius of the granule,

$$
v_{m}=4 \pi r^{2} \Delta
$$

and

$$
\frac{d n_{2}}{d \Delta}=-\frac{n_{2}^{4} n_{0}^{4}+n_{2}^{2} n_{0}^{2}-2}{6 n_{2} n_{0}^{2}} \cdot \frac{1}{\Delta}
$$

Taking $n_{0}=1.3450, n_{2}=1.107$ and $\Delta=90 \AA$, we obtain

$$
\frac{d n_{2}}{d \Delta}=-4.745 \times 10^{-3} \AA^{-1} \text {. }
$$

(B) Relation between $\frac{d S}{S}$ and $\frac{d \Delta}{\Delta}$

The geometrical cross-section of the granule is $S=\pi(r+\Delta)^{2}$. The relative change in the geometrical cross-section of the granule due to a relative change $\frac{d \Delta}{\Delta}$ in the thickness of the membrane (see Fig. 8) is given by:

$$
\frac{d S}{S}=\frac{2 \Delta}{r+\Delta} \frac{d A}{\Delta}
$$




\section{References}

Crowley, J.M. 1973. Electrical breakdown of bimolecular lipid membranes as an electrochemical instability. Biophys. J. 13:711

Douglas, W.W. 1965. Calcium-dependent links in stimulus-secretion coupling in the adrenal medulla and neurohypophysis. In: Wenner-Gren Center International Symposium, Series 5. U.S. von Euler, S. Rosell and B. Uvnäs, editors. p. 267. Pergamon Press, New York

Fenn, R.W., Oser, H. 1965. Scattering properties of concentric soot-water spheres for visible and infrared light. Appl. Optics. 4:1504

Grünhagen, H.H. 1974a. A high power square wave pulse generator for the investigation of fast electric effects in solution. Messtechnik 1:19

Grünhagen, H.H. 1974b. Fast spectrophotometric detection system for coupled physical and chemical electric field effects in solution. Biophysik 10:347

Hillarp, N.A. 1959. Further observations on the state of the catecholamines stored in adrenal medullary granules. Acta Physiol. Scand. 47:271

Ilgenfritz, G. 1966. Chemische Relaxation in starken elektrischen Feldern. Thesis. Univ. of Göttingen, Germany

Latimer, P., Moore, D.M., Bryant, F.D. 1968. Changes in total light scattering and absorption caused by changes in particle conformation. $J$. Theor. Biol. 21:348

Lowry, O.H., Rosebrough, N.J., Farr, A.L., Randall, R.J. 1951. Protein measurement with the Folin phenol reagent. J. Biol. Chem. 193:265

Neumann, E., Rosenheck, K. 1972. Permeability changes induced by electric impulses in vesicular membranes. J. Membrane Biol. 10:279

Rosenheck, K., Lindner, P., Pecht, I. 1975. Effect of electric fields on light scattering and fluorescence of chromaffin granules. J. Membrane Biol. 20:1

Smith, A.D. 1968. Biochemistry of adrenal chromaffin granules. In: The Interaction of Drugs and Subcellular Components in Animal Cells. P.N. Campbell, editor. p. 239. J. \& A. Churchill Ltd., London

Trifaró, J.M., Dworkind, J, 1970. A new simple method for isolation of adrenal chromaffin granules by means of an isotonic density gradient. Anal. Biochem. 34:403

Tsong, T.Y., Kingsley, E. 1975. Hemolysis of human erythrocytes induced by a rapid temperature jump. J. Biol. Chem. 250:786

van de Hulst, H.C. 1957. Light-Scattering by Small Particles. p.14. John Wyley, New York

Zimmerman, U., Pilwat, G., Riemann, F. 1974. Dielectric breakdown of cell membranes. Biophys. J. 14:881 